\title{
DIFFUSION COEFFICIENTS IN DENSE PLASMAS
}

\author{
G. Fontaine
}

and

\section{G. Michaud}

\author{
Département de Physique \\ Université de Montréal
}

It has been known for some time (Schatzman 1958) that diffusion could play an important role in the spectral evolution of white dwarfs. However, it is only recently that quantitative estimates have become available, making use of detailed envelope models (Fontaine and Michaud 1979, hereafter referred to as FM; Vaucla1r, Vauclair, and Greenstein 1979; Alcock and Illarionov 1979). These studies suggest that the observed monoelemental character of white dwarf spectra can qualitatively be explained in terms of the diffusion model. Moreover, it appears that the diffusion time scales are so short compared to evolutionary times that competing mechanisms, such as accretion, must be Invoked to explain the small but measurable abundances of heavy elements in the spectra of cooler white dwarfs.

Unfortunately, estimates of diffusion time scales under white dwarf conditions suffer from large uncertainties. First, if thermal diffusion dominates, the characteristic time scales strongly depend on the average charge of the diffusing ion. However, in cooler white dwarf envelopes, the ionization equilibrium is dominated by non-ideal effects, and it becomes very difficult to estimate the degree of ionization of a given element. Detailed numerical computations of the kind described by Fontaine, Graboske, and Van Horn (1977) must then be employed. Otherwise, as estimated by FM, the diffusion time scales of metals may be uncertain by factors $3-4$.

More importantly, however, is the fact that all estimates have so far been obtained from formulae derived by Chapman and Cowling (1970) which strictly apply in one limiting case: the plasma must be sufficiently dilute so that charged particles interact via a pure Coulomb potential. Using such a potential leads to a well-known divergence of the interaction cross-section which is removed with the introduction 
of an arbitrary long-range cutoff distance (usually taken as the Debye length). Such an approach has the main advantage that the cross-sections are expressable analytically; they have a logarithmic form such as equations (4) and (A4) of FM. A better approach is the use of screened Coulomb potentials. They permit, in principle, a better description of the collective effects of all the surrounding charges. They also remove the need for an ad hoc cutoff distance as the crosssection integrals converge riaturally, but the computations must be done numerically.

A second, non-essential, approximation used by Chapman and Cowling is to take the logarithmic term of the Coulomb cross-section outside of the integral over the velocity distribution on the assumption that the energy dependence of this logarithmic term would be weak. Again, this was done in order to obtain analytic expressions for the transport coefficients. The diffusion coefficient given, for exemple, by equation (3) of FM is the result of such an approximation. Numerical computations indicate, however, that systematic effects are introduced by such an approximation and that these effects may be large, even in main-sequence stars.

We have developed a computer code that numerically evaluates the Chapman-Cowling collision integrals for spherically symmetric potentials. The details of these calculations will be given elsewhere. We wish here to report on some preliminary calculations with special emphasis on white dwarfs. Table 1 gives both the diffusion coefficient $D_{12}$ and the thermal diffusion coefficient $\alpha_{12}$ of carbon diffusing in helium for a typical isotherm. Four cases are considered: CC is the result of applying directly the Chapman and Cowling equations; PC is for a pure Coulomb potential with Debye cutoff without taking the logarithmic term outside of the velocity distribution integral; DH stands for the Debye-HUlckel potential; TF is for the Thomas-Fermi potentlal which is a better representation of electron screening than the $\mathrm{DH}$ model at higher densities.

Comparing CC and PC gives the effects of taking the logarithmic term of the Coulomb cross-section outside of the velocity distribution integral. Comparing PC and DH leads to an estimate of the con-. sequences of having to choose an arbitrary cutoff distance. The most obvious feature is that the thermal diffusion coefficient, as estimated by $\mathrm{CC}$, strongly diverges to large positive values with increasing densities. By contrast, PC gives results for $\alpha_{12}$ which are quite close to those of $\mathrm{DH}$ up to $\log \rho \approx-2$, beyond which there is a divergence to large negative values. On the other hand, the screened potentials lead to small absolute values of $\alpha_{12}$ at high densities. It thus would appear that the thermal diffusion coefficient can be substantially overestimated if the Chapman-Cowling formulae are used. 
TABLE 1

Transport coefficients for $\log T=5.0(\mathrm{C} / \mathrm{He})$

\begin{tabular}{rcccccccr}
$\log \rho$ & \multicolumn{9}{c}{$\mathrm{D}_{12}$} & & \multicolumn{2}{c}{$\alpha_{12}$} & \\
& $\mathrm{CC}$ & PC & $\mathrm{DH}$ & $\mathrm{TF}$ & $\mathrm{CC}$ & $\mathrm{PC}$ & $\mathrm{DH}$ & $\mathrm{TF}$ \\
-10 & $9.76+4$ & $1.13+5$ & $1.20+5$ & -- & $1.49+1$ & $1.08+1$ & $1.00+1$ & -- \\
-8 & $1.34+3$ & $1.66+3$ & $1.82+3$ & -- & $1.52+1$ & $9.58+0$ & $9.14+0$ & - \\
-6 & $2.15+1$ & $3.09+1$ & $3.77+1$ & -- & $1.60+1$ & $7.12+0$ & $6.10+0$ & -- \\
-4 & $5.33-1$ & $1.10+0$ & $1.49+0$ & -- & $2.00+1$ & $2.52+0$ & $2.42+0$ & - \\
-2 & $8.64-2$ & $1.92-1$ & $1.55-1$ & $2.36-1$ & $1.48+2$ & $-3.28-1$ & $5.32-1$ & $2.64-1$ \\
0 & $7.85-2$ & $1.57-1$ & $3.71-2$ & $2.53-2$ & $1.26+4$ & $-4.15+0$ & $-7.77-3$ & $-1.79-1$ \\
2 & $7.85-2$ & $1.57-1$ & $1.43-2$ & $3.46-3$ & $1.25+6$ & $-3.44+2$ & $-1.81-1$ & $-3.55-1$
\end{tabular}

We also find that the diffusion coefficient $D_{12}$ tends toward a constant value with increasing densities in the $\mathrm{CC}$ and $\mathrm{PC}$ formalisms. By contrast, $\mathrm{D}_{12}$ keeps decreasing if the screened potentials are used. This means that in the denser, coolex white dwarfs, $\mathrm{D}_{12}$ is also overestimated if the Chapman-Cowling formulae are employed.

It is striking to note how $\alpha_{12}$ is much more affected than $D_{12}$ by the various approximations. It is easy to understand. While $\mathrm{D}_{12}{ }_{12}$ comes In a rather simple way from the collision probability, $\alpha,{ }_{12}$, the thermal diffusion coefficient, comes from a delicate cancellation of large and small velocity terms. Thermal diffusion is related to the energy flux when a temperature gradient is present (Cowling 1970). Because energy is flowing upwards, the upgoing protons, in the thermal distribution, will have more energy than the downgoing protons in a hydrogenrich star. If the element of interest has a larger cross-section for interacting with the upgoing protons it will be pushed upwards, downwards in the other case. This implies a very delicate cancellation which can be strongly affected by any approximation on an energy dependent term such as taking $A_{12}$ (see equation (4) of FM) out of the integral.

We have applied these considerations to reestimate the diffusion time scales of carbon and calcium in the helium-rich white dwarf envelopes discussed by FM. In figure 1, we compare the diffusion time scales computed by FM using the Chapman and Cowling formulae with the numerically-evaluated time scales obtained from the DH screened potential. The helium-rich envelope models used here are parametrized by $M / M_{Q}=0.612$ and $\log q=-5.0$, where $q$ is the fractional mass in the envelope.

It is most important to have reliable estimates of the diffusion time scales for cooler white dwarfs since metals are seen only in the spectra of these stars. Figure 1 , however, indicates that for $T_{e}<20000 \mathrm{~K}$, the CC formulae lead to substantial underestimates of the the characteristic time scales. Detailed calculations are therefore needed at the low-temperature end of the white dwarf cooling sequence. 


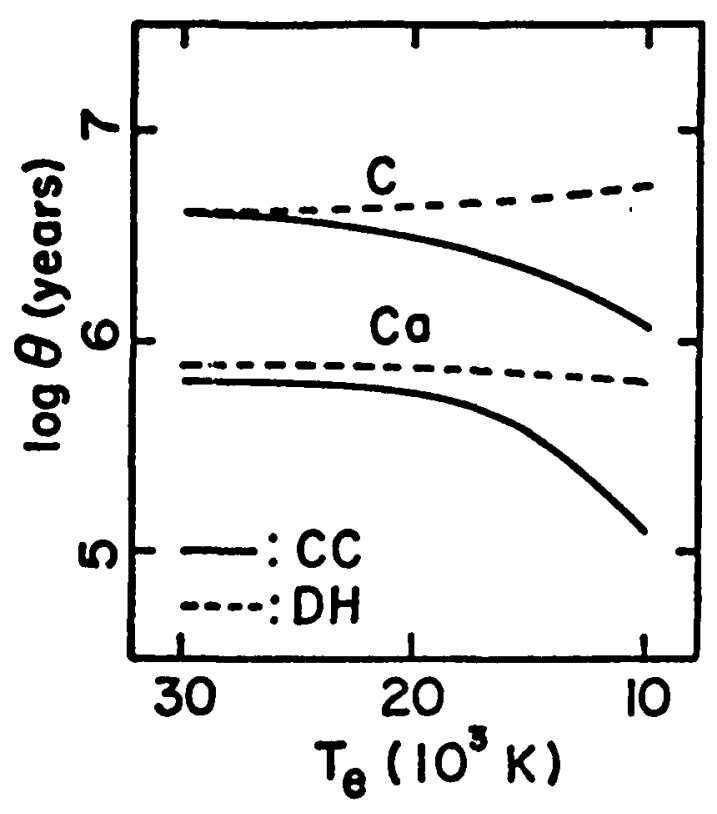

Fig. 1. Diffusion Time Scale

vs Effective Temperature

The diffusion time scales estimated here are still much smaller than the evolutionary times so that the central conclusion of FM is left unchanged: if allowed to proceed freely without competition, diffusion leads to completely pure hydrogen or helium white dwarf atmospheres. We note, however, that before quantitative comparisons can be made with the observed abundances of heavy elements in the spectra of cooler white dwarfs, calculations such as those reported here will be needed. In particular, estimates of equilibrium abundances of heavy elements based on a balance between accretion and diffusion have to take into account the large uncertainties in both the accretion rate and the diffusion time scale.

It should be noted that the preliminary results presented here yet suffer from another uncertainty. Indeed, thermal diffusion not only depends on $\alpha_{12}$ but also on $\alpha_{1 e}$, the thermal diffusion coefficient of electrons refative to the maln ionic species. We have not yet solved all the numerical problems in obtaining $\alpha_{\text {f }}$ for screened potentials. In calculating the time scales of figure $t$, we used in all cases the $\alpha_{1 \text { e }}$ of as given by equation (A3) of FM. It is not clear at the moment whether $\alpha$ will be positive or negative! The changes indicated on figure 1 coutid actually be larger. In particular, the slight electron degeneracy that is beginning to appear at the bottom of the convection zone may play an important role. Indeed, at this stage, it is not completely excluded that it could stop diffusion and eliminate the need for accretion. 
As a final remark, we wish to stress that while our approach takes Into account screening effects in a more correct way, it is based on the Chapman-Enskog solution of the Boltzmann equation and does not apply at very large densities. Rigorous statistical mechanical results based on molecular dynamics simulation are becoming available (DeWitt 1976, 1977; Vieillefosse and Hansen 1975; Hansen 1977). Unfortunately, these estimates of transport coefficients are valid only at relatively high densities where the one-component plasma model applies. Thus the electrons must be strongly degenerate so as to provide a uniform neutralizing background, and the ions must be strongly correlated. Except for the coolest white dwarfs these conditions are encountered only much deeper than the base of the convection zone so that these results cannot be directly applied to the problem of the spectral evolution of white dwarfs. However, it is not clear at the moment exactly where one should shift from one regime to the other.

\section{REFERENCES}

Alcock, C., and Illarionov, A. 1979, preprint.

Chapman, S., and Cowling, T.G. 1970, "The Mathematical Theory of NonUniform Gases" (Cambridge: Cambridge University Press).

Cowling, T.G. 1970, J. Phys. A., $\underline{3}, 774$.

DeWitt, H.E. 1976, Phys. Rev. A, 14, 1290.

- 1977, NATO Summer Institute on Strongly Coupled Plasmas, Orléans-1a-Source, France.

Fontaine, G., Graboske, H.C.Jr., and Van Horn, H.M. 1977, Ap. J. Suppl., $\underline{35}, 293$.

Fontaine, G., and Michaud, G. 1979, Ap. J. (in press)。

Hansen, J.-P. 1977, NATO Summer Institute on Strongly Coupled Plasmas, Orléans-la-Source, France.

Schatzman, E. 1958, "White Dwarfs" (Amsterdam: North-Holland).

Vauclair, G., Vauclair, S., and Greenstein, J.L. 1979, preprint.

Vieillefosse, P., and Hansen, J.-P. 1975, Phys. Rev. A, 12, 1106. 